\title{
External Force Observer for Medium-sized Humanoid Robots
}

\author{
Louis Hawley and Wael Suleiman
}

\begin{abstract}
In this paper, we introduce a method to estimate the magnitude of an external force applied on a humanoid robot. The approach does not require using force/torque sensors but instead uses measurements from commonly available forcesensing resistors (FSR) inserted under the feet of the humanoid robot. This approach is particularly interesting for affordable medium-sized humanoid robots such as Nao and Darwin-OP. The main idea is to use a simplified dynamic model of a linear inverted pendulum model (LIPM) subjected to an external force, and the information from the robot inertial measurement unit (IMU) and FSR sensors.

The proposed method was validated on a Nao humanoid robot to estimate the external force applied in the sagittal plane through two experimental scenarios, and the results pointed out the efficiency of the proposed observer.
\end{abstract}

\section{INTRODUCTION}

Humanoid robots are good candidates to perform manipulation and transport tasks since they possess articulated arms. These tasks require the robot to be able to adjust its gait in order to take into account the external forces exerted on it. In such situations, the robot usually uses his interoceptive/exteroceptive sensors to estimate those forces and a representative dynamic model to generate stable patterns.

A situation in which the knowledge of the transported mass or applied force could be very useful to generate a more stable gait is the transportation of an object on a cart by a robot. This scenario is considered in [1], where a motion planner uses different sets of motion primitives depending on the mass transported on the cart. The estimation of the load is done by making the robot execute a turning in place motion and by looking at the error between the planned motion and the actual robot position. Although this approach can effectively differentiate a light load from a heavy one, the differentiation is done by roughly applying a binary operator to the error. The planning algorithm then chooses the heavy load or small load primitives set accordingly. A better estimation of the load could allow a more optimal motion planning as more primitive sets adapted to different load could be used. Moreover, by integrating the estimated external force into the pattern generation module, more stable motions can be obtained even with a relatively heavy load.

\section{A. Relevant works}

Improving the robustness of humanoids walk against disturbance is a topic of interest since these robots are expected to perform tasks in a variety of human environments. In [2], a disturbance observer that estimates the magnitude of an

Louis Hawley and Wael Suleiman are with Electrical and Computer Engineering Department, the Faculty of Engineering, University of Sherbrooke, Sherbrooke, Canada \{louis.hawley, wael.suleiman\}@usherbrooke.ca

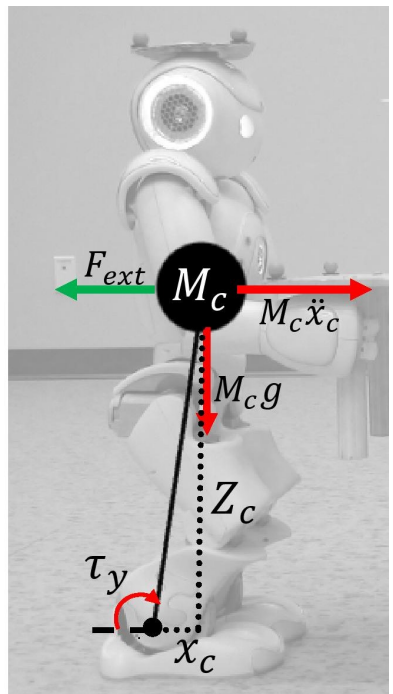

Fig. 1. Simplified model of a humanoid robot with an external force

external force is presented. The observer uses measurement from an IMU and six-axis force/torque (F/T) sensors located at the ankles to detect and estimate the magnitude of a strong force such as a kick to the chest of the robot or a collision with the environment. Whereas a kick to the chest can be represented by an impulse input applied to the system, a pushing motion is equivalent to a step input and it is therefore expected that the observer would not have the same performance in the latter case.

Some research work were also conducted to estimate an external force applied on a small humanoid robot. In [3] and [4], the authors are interested in predicting the perturbations transmitted to a robot in a human-robot interaction. Essentially, the proposed approach is to generate a probabilistic model of the sensors output to predict future readings. Then, if the measurements are not in accordance with the model, it can be concluded that an external force is applied. The authors were able to use the system in a humanrobot interaction to infer the human intention and move the robot accordingly. However, in a robot-human interaction, the robot does not need to modify its walking gait since the human will apply more or less force depending on the robot reaction. But if the humanoid is pushing or transporting an object, it must adjust its gait to remain stable. In this case, an estimation of the force magnitude would be necessary.

In [5], [6], the ZMP dynamic is analysed for a humanoid robot performing a pushing manipulation task. In this case, the external force is directly measured through $\mathrm{F} / \mathrm{T}$ sensors located in the wrist of a HRP-2 robot. Their results reveal 
that the robot falls if the motion generator does not consider the exerted force on the grippers. On the other hand, the required compensation can be extracted by computing two ZMPs. The first ZMP, referred as the generalized ZMP, is computed by considering the gravity and the reaction force and moment on the floor. A second ZMP is computed by considering the external force applied on the grippers. The difference between those two ZMPs is added to the desired ZMP trajectory, which is generated using the LIPM model. This procedure was successfully applied in simulation to generate a stable motion. There are also numerous works [?] [?] where the ZMP position error is monitored and integrated into the control law. In these cases, quantifying the perturbation and determining its origin were not addressed. As the ZMP was successfully used in all these previous work to make humanoid walk more robustly against disturbance, a plausible approach to approximate an external force would be monitoring the ZMP variation and linking it to an external force. The objective is then to have a dynamic model of the task being executed and to be able to measure the actual ZMP with enough precision.

In this work, assuming the robot is equipped with forcesensing resistors (FSR) under the feet, we propose a method for estimating the magnitude and direction of an external force in the sagittal plane using the LIPM model and ZMP measurements using the FSR. The proposed observer was designed for and validated on a Nao humanoid robot. Nao [7] is a medium-sized humanoid robot manufactured by Aldebaran. On the contrary of complex and highly sophisticated humanoid robots, such as HRP-2 or Atlas, Nao does not have six-axis F/T sensors and possesses only FSR under the feet.

The LIPM model and the dynamic equations with an external force are presented in Section II. Section III deals with estimating the ZMP using the available sensors. The external force-observer architecture and implementation is addressed in section IV. Finally, in section V, experimental results are presented and the observer performance is analyzed and discussed.

\section{DYNAMIC MODELS}

\section{A. LIPM dynamic}

LIPM model has been widely used to generate stable walking patterns [8]. According to this model, the Center of Mass (CoM) only moves under the action of the gravity. The dynamics of the LIPM can be decoupled within each axis and therefore we only show hereafter the equation in the sagittal plane. The motion dynamic can be written as

$$
M_{c} \ddot{x}_{c}=\frac{M_{c} g x_{c}}{Z_{c}}
$$

where $M_{c}$ is the mass of inverted pendulum, $Z_{c}$ is the height of CoM, $g$ is the magnitude of gravity acceleration, $x_{c}$ and $\ddot{x}_{c}$ are the position and the acceleration of the projection of CoM on the sagittal axis. Note that $x$ is expressed in the pivot frame, which corresponds to the ankle on the real system.

\section{B. Dynamic model with external force}

The general dynamic model of a robot walking with an external force applied is presented in Fig. 1, and is defined as follows:

$$
M_{c} \ddot{x}_{c}=\frac{M_{c} g x_{c}}{Z_{c}}-F_{\text {ext }}
$$

where $F_{\text {ext }}$ is an external force. For instance, this force might be the result of the robot pushing/lifting an object or interacting/collaborating with a human.

Here, we have a system identification problem where we need to estimate $F_{e x t}$ in order to generate a stable motion. Although monitoring $\ddot{x}_{c}$ or $x_{c}$ might give us an insight into the external force that is applied, it would not be very useful in the case of a position-controlled robot such as Nao. Unless the external force is strong enough that the motors of the robot are unable to keep their position, the CoM position will not be affected. However, it is worth mentioning that a strong punctual disturbance such as a push will affect the CoM position and acceleration as it is the main idea behind the external force observer in [2].

1) $Z M P$ without external force $\left(x_{Z M P}^{\prime}\right)$ : First, we consider the ZMP according to its general definition for a humanoid robot, approximated by a LIPM, without external force. In other words, we only consider the gravity and the inertial force of the robot. Recalling that the ZMP is a point on the ground where the inertial and gravity moments cancel out, it is defined as

$$
x_{Z M P}^{\prime}=x_{c}-\frac{Z_{c} \ddot{x}_{c}}{g}
$$

We will refer to the ZMP computed without considering the external force as $x_{Z M P}^{\prime} \cdot x^{\prime} Z M P$ can be directly obtained from the desired ZMP trajectory. It can also be computed using the acceleration from the IMU to approximate $\ddot{x}_{c}$ and the direct kinematic to get $x_{c}$.

2) ZMP: The true ZMP can be found by considering every force acting on the system. From (2), the ZMP is defined as:

$$
x_{Z M P}=x_{c}-\frac{Z_{c} \ddot{x}_{c}}{g}-\frac{F_{e x t} Z_{c}}{M_{c} g}
$$

We will refer to the ZMP computed considering the external force as $x_{Z M P}$. The basic idea of our external force observer is that the difference between the actual ZMP $\left(x_{Z M P}\right)$ and the planned ZMP $\left(x_{Z M P}^{\prime}\right)$ is proportional to the external force. The main challenge is then to get a good estimate of the actual ZMP. The next section deals with estimating the ZMP with measurements from the available sensors.

\section{ZMP ESTIMATION}

As presented in [9], the ZMP can be estimated with force-sensing resistors located under the feet. The main idea is that by measuring the applied force on each force sensor, it is possible to estimate the center of pressure (CoP) 
by calculating the position of the equivalent force. Then, recalling that the $\mathrm{ZMP}$ and the $\mathrm{CoP}$ are the same point if the robot is in a stable configuration [10], we can estimate the ZMP by computing the CoP

$$
x_{Z M P}=x_{C o P}
$$

\section{A. Center of pressure measurements}

Each foot of Nao possesses 4 FSR that each returns a force between 0 and $25 \mathrm{~N}$. .Using these measurements, one can easily compute the center of pressure on one foot as

$$
x_{C o P}=\frac{\sum_{i=1}^{4} F_{i} x_{i}}{\sum_{i=1}^{4} F_{i}}=\frac{\sum_{i=1}^{4} F_{i} x_{i}}{F_{T}}
$$

where $F_{i}$ is the force measured at the $i^{\text {th }} \mathrm{FSR}, x_{i}$ is the position of the $i^{\text {th }}$ FSR in the foot frame and $F_{T}$ is the total force applied on the foot.

During walking, we assume that the robot is always in a single support phase. Therefore, the support foot must be detected to apply the previous formula. A possible solution to determine the support foot is to consider the total force applied on each foot (measured with the FSR) as the decision variable. However, using a simple boolean operator results into false support foot detection since the force measured by the FSR becomes highly noisy when a foot lands on the floor.

The adopted solution, similar to [11], is to implement a Schmitt trigger to process the support foot state. The Schmitt trigger uses a low and a high threshold to determine the value of the output. In our case, the input to the trigger is the total force on the right foot minus the total force on the left foot. Hence, positive value means that the support foot is the right and a zero value means the support foot is the left. Satisfactory results were obtained by setting the positivegoing threshold at $2 N$ and the negative-going threshold at $-2 N$.

However, when the robot is immobile and in doublesupport mode, the ZMP (or CoP) can be simply found using the following formula

$$
x_{C o P}=\frac{x_{C o P}^{l} F_{T}^{l}+x_{C o P}^{r} F_{T}^{r}}{F_{T}^{l}+F_{T}^{r}}
$$

where $x_{C o P}^{*}$ and $F_{T}^{*}$ are computed as in (6) for the left and right feet.

\section{B. External force observer}

From (3) and (4), the external force $F_{\text {ext }}$ can be easily found

$$
F_{\text {ext }}=M_{c} g \frac{\left(x_{Z M P}^{\prime}-x_{Z M P}\right)}{Z_{c}}
$$

where $x_{Z M P}^{\prime}$ is the ZMP computed using the LIPM model and $x_{Z M P}$ is measured with the FSR.

\section{IMPLEMENTATION}

The architecture of the observer is summarized in Fig. 2. Implementation details are presented in the following subsections.

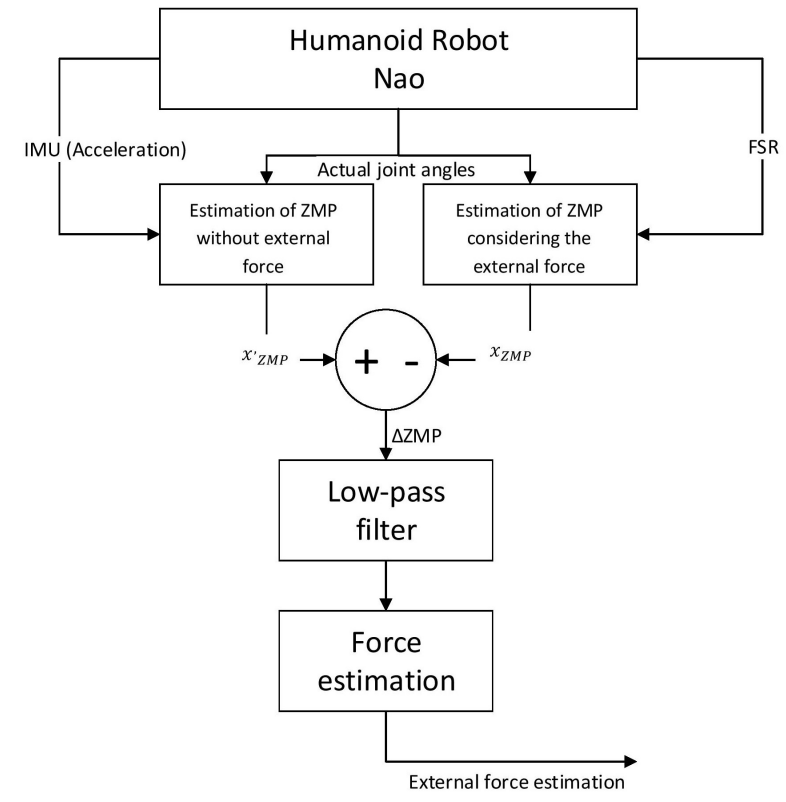

Fig. 2. Block diagram of the observer

\section{A. $x^{\prime}{ }_{Z M P}$ Computation}

As mentionned before, $x_{Z M P}^{\prime}$ corresponds to the position at which the ZMP would be if no external force is applied. For humanoid robots that use a ZMP-based control scheme to generate the walking gait, it typically corresponds to the desired ZMP trajectory. However, to compute the ZMP with (3) the position and acceleration of the CoM must be approximated.

1) CoM acceleration: The acceleration of the CoM can be extracted from the IMU, which is a standard part of a humanoid robot sensors. As for Nao, the IMU provides measurements from a three-axis accelerometer and a twoaxis gyroscope. Also, an existing on-board algorithm provides an estimation of the torso orientation. In this work, we make the assumption that the CoM coincides with the IMU. Therefore, incoming data from the accelerometer can be used to approximate the acceleration of the CoM. Fig. 3 presents a block diagram of the raw acceleration data processing. The projection into an inertial frame and the gravity compensation steps are implemented as presented in [12].

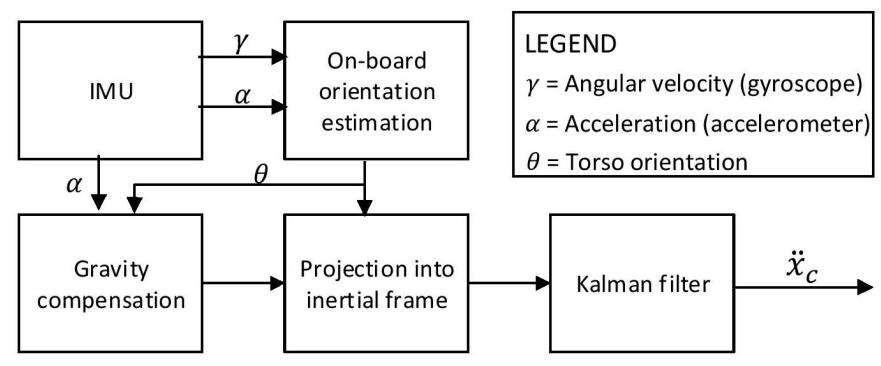

Fig. 3. Block diagram of the processing done on the raw acceleration data 
2) CoM Position: In order to determine the CoM position, we make the assumption that the trunk of Nao is the center of mass. This assumption is also made in the built-in walk engine of Nao as presented in [13]. Therefore, simple direct kinematic computation with encoder readings from the servo motors is used to determine the position of the CoM in the support foot frame. The support foot is determined using the method presented in Section III-A.

\section{B. Low-pass filtering (Sway motion cancellation)}

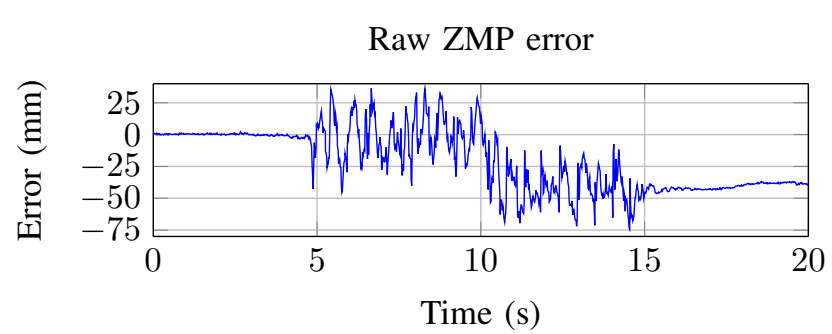

Filtered ZMP error

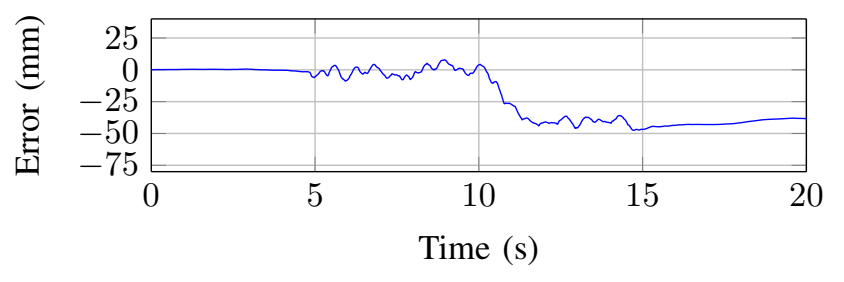

Fig. 4. ZMP error during a sagittal walk before and after filtering. At $T=10 \mathrm{~s}$, a constant force was applied

During the walk, the torso of a humanoid robot will naturally oscillate in what is typically called the sway motion. Obviously, the ZMP will also oscillate in the same manner. If this swaying oscillation is not removed at some point, it will highly degrade the performance of the observer. This problem has already been tackled in [14], where the filtering of the Nao robot sway motion is analysed. It is mentionned that the frequency of the sway motion for the Nao robot is close to $1 \mathrm{~Hz}$. This claim was validated on our robot, and a first order low-pass filter with a cut-off frequency of $0.6 \mathrm{~Hz}$ has been used. Fig. 4 presents the error signal between the two ZMPs before and after filtering during a sagittal walk.

\section{EXPERIMENTAL RESULTS}

The observer was tested in two scenarios : stationary robot with an external force and a walking robot with an external force. All the experiments results were generated with the following parameters: $g=9.81 \mathrm{~m} / \mathrm{s}^{2}, Z_{c}=0.315 \mathrm{~m}$, sampling period $(T)=16.7 \mathrm{~ms}$ and $M_{c}=4.5 \mathrm{Kg}$.

\section{A. Case Study 1 : Stationary with external force}

The dynamic model of the first scenario considered is presented in Fig. 6. The used setup for this experiment is presented in Fig. 5. In this experiment, the robot is standing still and attached from the waist to a mass in the form of a water bottle. At some point, the mass is released and the force propagates to the robot through a basic pulley system.

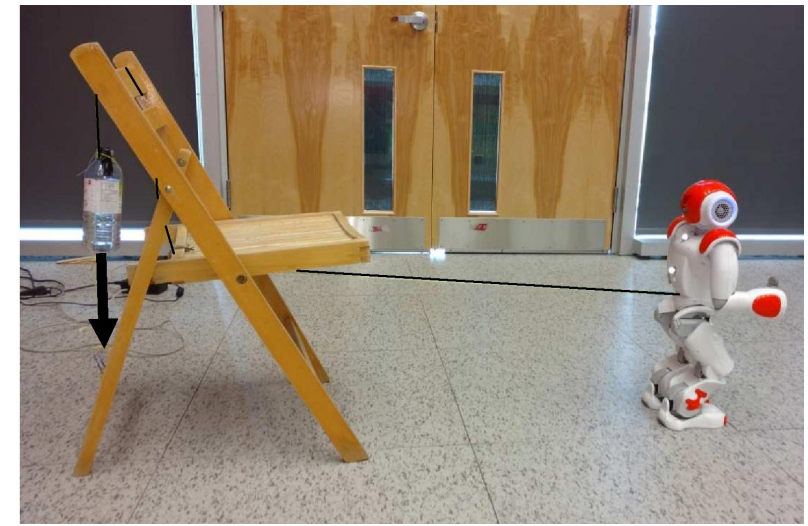

Fig. 5. Experimental setup used to apply a known external force

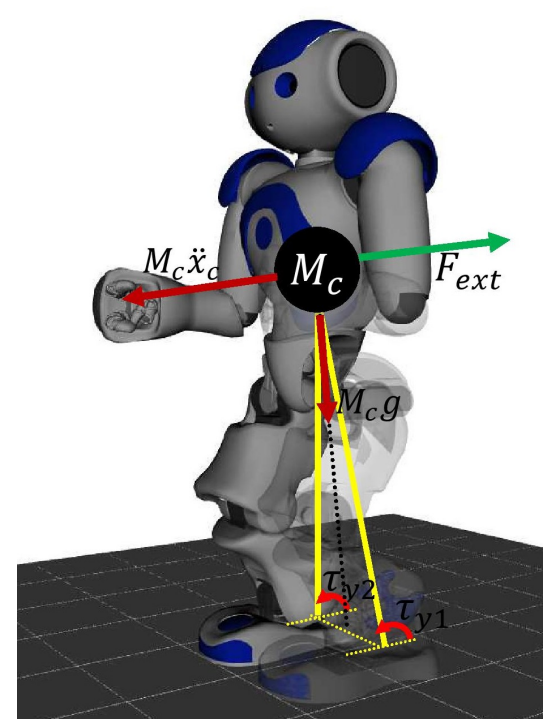

Fig. 6. Case study $1:$ stationary robot with an external force

1) Results: Fig. 8 presents the results of an experiment where a constant force of approximately $3.8 \mathrm{~N}$ was applied on a static robot. Fig. 8(a) shows that the external force has no significant effect on the CoM acceleration and that the servo motors are able to keep their position despite the added force since the $x_{Z M P}^{\prime}$ is not affected. On the other hand, the ZMP measured with the FSR is rapidly shifted by more than $3 \mathrm{~cm}$. In Fig. 8(b), the difference between the ZMPs is presented after low-pass filtering. Since the robot is not walking, the filter presented in Section IV-B is not necessary here. Instead, a low-pass filter with a cut-off frequency of 4 $\mathrm{Hz}$ was used to smooth the signal. Fig. 8(c) shows that the observer estimated force is close to the actual one. In order to characterize the performance of the observer, we define the settling time as the time needed for the output to reach and stay within a $20 \%$ margin of the reference value. In this case, the settling time is close to $0.5 \mathrm{~s}$. Similar results were obtained in experiments performed with external force ranging from $1 \mathrm{~N}$ to $4 \mathrm{~N}$. In each case, the observer was able to estimate the external force within a $20 \%$ error margin. 


\section{B. Case Study 2 : Walking with external force}

The experimental setup used in this case is the same as in Case 1 (Fig. 5). Initially, the robot is standing still and attached from the waist to a mass which is in full contact with the ground. The robot then starts to walk forward and at some point, the rope is completely tensed and the mass is lifted off the ground by the walking robot, as shown in Fig. 7. The lifted mass acts as an external force pulling the robot backwards.

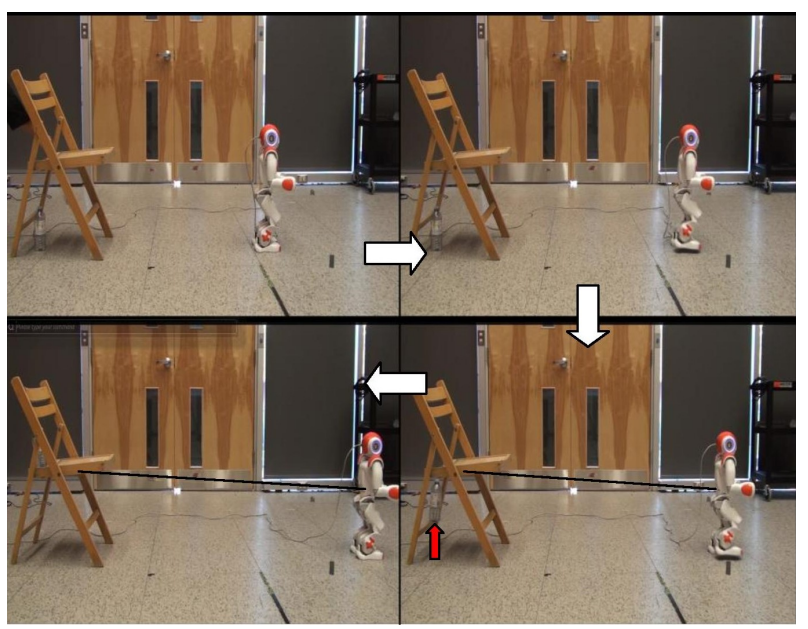

Fig. 7. Snapshots of an experiment where a mass is lifted off the floor by a Nao robot walking forward

1) Results: The experimental results of a forward walk with an external force of $3 \mathrm{~N}$ is presented in Fig. 9. As shown in Fig.9(a), the two ZMPs are similar until the external force is applied at around 10s. At this point, the $x_{Z M P}$ is shifted to the back as opposed to the $x_{Z M P}^{\prime}$ that continues to oscillate around 0. However, as demonstrated in Fig. 9(b), there is a small error between the two ZMPs even when no force is applied. Thus, a threshold operation is applied on the error signal to avoid detecting a false external force. Accordingly, the observer output is more stable but external forces of magnitude less than $0.5 \mathrm{~N}$ are not detected. In Fig. 8(c), the observer estimated force is given along with the true external force. One can figure out that the observer successfully estimated the true external force. Also, as might be seen, the settling time is slightly more than $1 \mathrm{~s}$. In this experiment, it corresponds to two walking steps for the robot.

During similar experiments, the observer was able to estimate an external force ranging from $0.8 \mathrm{~N}$ to $3.8 \mathrm{~N}$ within a $20 \%$ error margin. Note that the maximum force that we could apply on the walking Nao, using the robot built-in walk engine, without making it fall, was $3.8 \mathrm{~N}$.

\section{CONCLUSION}

In this paper, we introduced a method to estimate the magnitude of an external force acting on a humanoid robot without using expensive 6-axis force/torque sensors. Essentially, it uses measurement from force-sensing resistors located under the feet of the robot to estimate the position of the ZMP and compare it to a reference ZMP that is computed using the linear inverted pendulum model. This approach is mainly interesting for medium-sized humanoid robots, and it was successfully validated on a Nao robot in two different scenarios.

Future work will focus on integrating the external force observer into the pattern generation module.

\section{ACKNOWLEDGMENT}

This research is supported by the Natural Sciences and Engineering Research Council of Canada (NSERC).

\section{REFERENCES}

[1] A. Rioux and W. Suleiman, "Humanoid Navigation and Heavy Load Transportation in a Cluttered Environment," in IEEE International Conference on Intelligent Robots and Systems (IROS), 2015, pp. 2180 2186

[2] K. Kaneko, F. Kanehiro, M. Morisawa, E. Yoshida, and J.-P. Laumond, "Disturbance observer that estimates external force acting on humanoid robots," 12th IEEE International Workshop on Advanced Motion Control (AMC), pp. 1-6, 2012.

[3] E. Berger, M. Sastuba, D. Vogt, B. Jung, and H. Ben Amor, "Dynamic Mode Decomposition for perturbation estimation in human robot interaction," The 23rd IEEE International Symposium on Robot and Human Interactive Communication, pp. 593-600, 2014. [Online]. Available: http://ieeexplore.ieee.org/articleDetails.jsp?arnumber $=6926317$

[4] E. Berger, D. Vogt, N. Haji-Ghassemi, B. Jung, and H. B. Amor, "Inferring guidance information in cooperative human-robot tasks," IEEE-RAS International Conference on Humanoid Robots, vol. 2015February, no. February, pp. 124-129, 2015.

[5] K. Harada, S. Kajita, K. Kaneko, and H. Hirukawa, "ZMP Analysis for Arm / Leg Coordination," in IEEE/RSJ International Conference on Intelligent Robots and Systems, 2003, pp. 75-81.

[6] _ _Pushing manipulation by humanoid considering two-kinds of ZMPs," IEEE International Conference on Robotics and Automation, pp. 1627-1632, 2003.

[7] D. Gouaillier, V. Hugel, P. Blazevic, C. Kilner, J. Monceaux, P. Lafourcade, B. Marnier, J. Serre, and B. Maisonnier, "Mechatronic design of NAO humanoid," IEEE International Conference on Robotics and Automation, pp. 769-774, 2009.

[8] S. Kajita and K. Tani, "Experimental study of biped dynamic walking in the linear inverted pendulum mode," IEEE International Conference on Robotics and Automation, vol. 3, pp. 2885-2891, 1995.

[9] B. G. Shim, E. H. Lee, H. K. Min, and S. H. Hong, "Study of Zmp Measurement for Bipedrobot Using Fsr Sensor," in 21st International Symposium on Automation and Robotics in Construction, 2004.

[10] M. Vukobratović and B. Borovac, "Zero-Moment Point - Thirty Five Years of Its Life," International Journal of Humanoid Robotics, vol. 01, no. 01, pp. 157-173, 2004.

[11] X. Xinjilefu, S. Feng, and C. G. Atkeson, "Center of Mass Estimator for Humanoids and its Application in Modelling Error Compensation, Fall Detection and Prevention," IEEE-RAS International Conference on Humanoid Robots, pp. 67-73, 2015.

[12] M. Bellaccini, L. Lanari, A. Paolillo, and M. Vendittelli, "Manual guidance of humanoid robots without force sensors: Preliminary experiments with NAO," IEEE International Conference on Robotics and Automation, pp. 1184-1189, 2014.

[13] D. Gouaillier, C. Collette, and C. Kilner, "Omni-directional closedloop walk for NAO," 10th IEEE-RAS International Conference on Humanoid Robots, pp. 448-454, 2010.

[14] G. Oriolo, A. Paolillo, L. Rosa, and M. Vendittelli, "Vision-based trajectory control for humanoid navigation," IEEE-RAS International Conference on Humanoid Robots, pp. 118-123, 2015. 


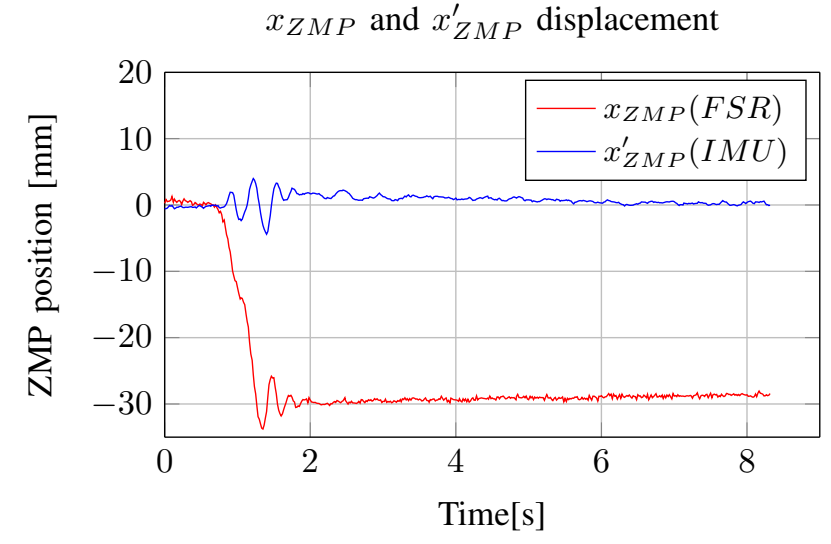

(a)

Filtered $\Delta \mathrm{ZMP}$

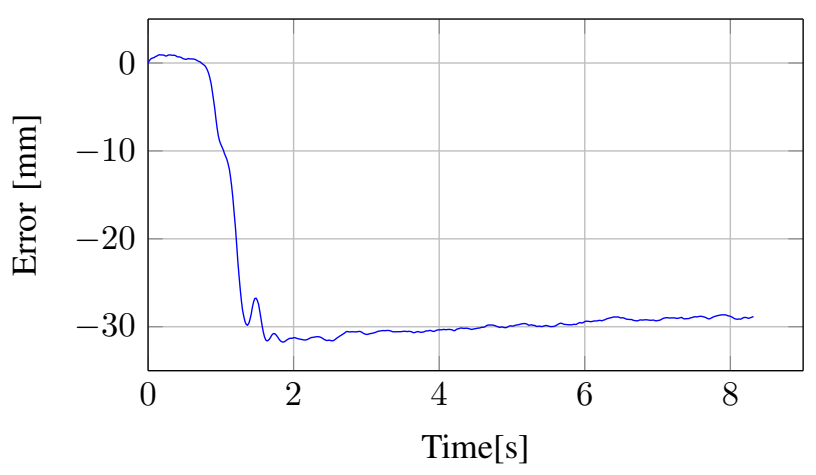

(b)

Estimated external force

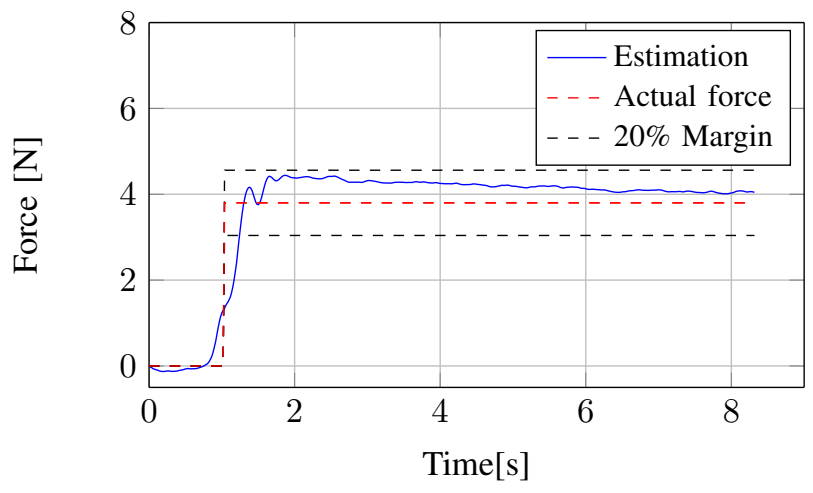

(c)

Fig. 8. Case Study 1: ZMP variation and force estimation when an external force of approximately $3.8 \mathrm{~N}$ is pulling a stationary Nao backward At approximately 1 second, the force is applied. (a) The displacement of $x_{Z M P}$ and $x_{Z M P}^{\prime}$ when the force is applied (b) The difference between the two ZMPs after applying a low-pass filter (c) The actual external force and the observer estimation

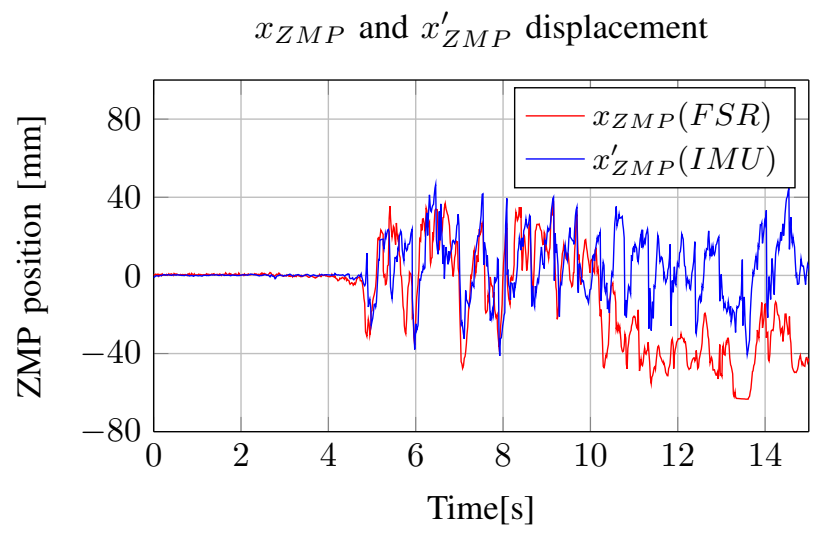

(a)

Filtered $\triangle Z M P$

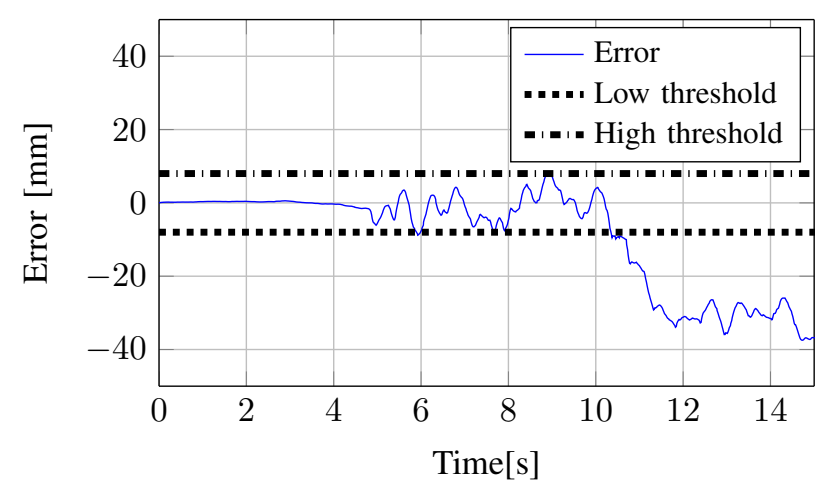

(b)

Estimated external force

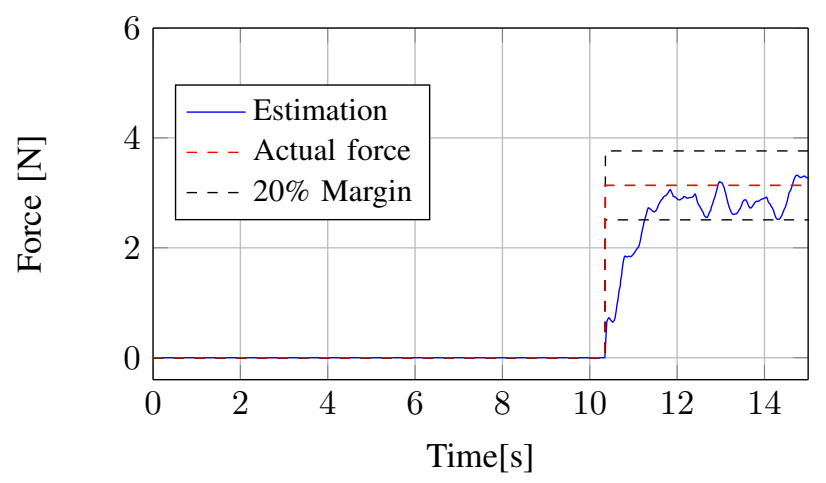

(c)

Fig. 9. Case Study 2: ZMP variation and force estimation when an external force of approximately $3 \mathrm{~N}$ is applied on a walking Nao. At approximately 10 seconds, the force is applied. (a) The displacement of $x_{Z M P}$ and $x_{Z M P}^{\prime}$ when the force is applied (b) The difference between the two ZMPs after applying a low-pass filter (c) The actual external force and the observer estimation 